\title{
Nível de atividade física em adolescentes e sua associação com variáveis sociodemográficas
}

\author{
Alex Pinheiro Gordia \\ Teresa Maria Bianchini de Quadros \\ Wagner de Campos \\ Édio Luis Petroski \\ https://doi.org/10.5628/rpcd.10.01.172
}

\author{
Centro de Pesquisa em Exercício e Esporte \\ Universidade Federal do Paraná \\ Curitiba, Paraná \\ Brasil \\ Núcleo de Pesquisa em Cineantropometria \\ e Desempenho Humano \\ Universidade Federal de Santa Catarina \\ Florianópolis, Santa Catarina \\ Brasil
}

\begin{abstract}
RESUMO
O presente estudo objetivou avaliar o nível de actividade física (NAF) de adolescentes, bem como investigar sua associação com variáveis sociodemográficas. Participaram desta investigação 608 estudantes de 14 a 20 anos de idade, de ambos os sexos, do ensino médio do município da Lapa, Paraná. O NAF foi estimado através do IPAQ e a condição socioeconômica foi avaliada de acordo com o Critério de Classificação Econômica do Brasil. Inicialmente, recorreu-se à análise descritiva, em seguida, foram empregados os testes do Qui-quadrado, teste t de Student e ANOVA one-way para analisar diferenças, a Regressão Logística foi utilizada para examinar as associações. O NAF dos adolescentes foi alto $(76,8 \%)$ ou moderado $(20,9 \%)$ e apenas $2,3 \%$ apresentaram NAF baixo, com gasto energético médio de $7039 \pm 5602$ MET-minuto/semana. As moças tiveram 1,6 vezes mais chance de apresentar NAF baixo quando comparadas aos rapazes. Os adolescentes do turno matutino tiveram 2,2 vezes mais chance de apresentar NAF baixo em relação àqueles do turno noturno. Com base nestes achados, programas de promoção da saúde e incentivo à prática de atividade física regular devem considerar o sexo e o turno de estudo como vaiáveis chave para mudanças no NAF de adolescentes.
\end{abstract}

Palavras-chave: estilo de vida, atividade física, fatores sociodemográficos, adolescente

\section{ABSTRACT \\ Physical activity Level in adolescents and its association with sociodemographic variables}

This study aimed to evaluate physical activity level in adolescents and to investigate its association with sociodemographic variables. A total of 608 adolescents (14-20 years), of both sexes, of the high school from Lapa, Paraná, Brazil participated in the study. Their PAL was estimated using the IPAQ, and their socioeconomic status was assessed according to the Brazilian Economic Classification Criteria. Initially, a descriptive analysis was carried out, followed by the Chi-square test, Student's $t$ test and one-way ANOVA to analyze differences and Logistic Regression to examine associations. The PAL of these adolescents was high (76.8\%) or moderate (20.9\%) and just $2.3 \%$ had a low PAL, with mean energy expenditure of 7039 \pm 5602 METminute/week. The girls had a 1.6 times greater chance of exhibiting a low PAL when compared with the boys. Adolescents who attended school during the morning had 2.2 times greater chance of having a low PAL than those who attending in the evenings. Health promotion programs encouraging the regular practice of physical activity should take account of sex and whether the students study in the mornings or evenings as being key variables to changing the PAL of adolescents.

Key-words: lifestyle, physical activity, sociodemographic factors, adolescents 


\section{INTRODUÇÃO}

Pesquisas recentes apresentam uma consistente relação inversa entre a atividade física e doenças crônico-degenerativas em indivíduos adultos, como doenças cardiovasculares(20), câncer de cólon em mulheres(31) e doença da vesícula biliar(18), bem como relação positiva com a qualidade de vida(32). Em adolescentes, diversos estudos observam a associação da atividade física com fatores de risco cardiovascular(1), síndrome metabólica(24) e obesidade(26). Estes achados são preocupantes tendo em vista que a presença de disfunções metabólicas na infância e adolescência aumenta o risco para morbi-mortalidade precoce na vida adulta(10,11). Além disso, uma recente revisão sistemática sobre a atividade física na adolescência demonstrou que há evidências consistentes de que indivíduos ativos durante a adolescência apresentam maior chance para tornarem-se adultos ativos(10). Diversos fatores têm se apresentado como determinantes do nível de atividade física (NAF) durante a adolescência, em especial variáveis sociodemográficas, como sexo, condição socioeconômica, escolaridade, educação materna ao nascimento, ordem de nascimento, local de moradia e tipo de escola(11, 12, 21, 22, 27). Entretanto, estudos realizados em diferentes regiões têm comumente apresentado resultados contrastantes $(11,12,21,22,27)$, indicando a necessidade de maiores investigações sobre a associação do NAF com fatores sociodemográficos na adolescência, visando a identificação de variáveis preditivas da inatividade física.

Há evidências de que o turno de estudo é uma variável sociodemográfica relevante para a condição de saúde e adoção de comportamentos (saudáveis ou não) em adolescentes $(5,6)$, contudo, pouco se sabe sobre a associação desta variável com o NAF de jovens. Estudo recente desenvolvido por Tenório et al.(30) avaliou a associação do turno de estudo com a inatividade física em adolescentes estudantes da rede pública estadual de ensino médio do Estado de Pernambuco, Brasil. Os achados do estudo indicaram que adolescentes que estudavam no turno noturno apresentaram menor chance de exposição ao comportamento sedentário em comparação com seus pares do turno diurno. No entanto, considerando a regionalidade da amostra investigada, os autores recomendam a realização de outras pesquisas para confirmação dos achados e avanço do conhecimento sobre a relação da atividade física com o turno de estudo na adolescência.

Desta forma, evidencia-se a necessidade de investigar a prevalência de sedentarismo e fatores sociodemográficos associados ao NAF em adolescentes para que intervenções, ainda nesta fase, sejam realizadas com sucesso, principalmente porque a adolescência representa um período crítico, no qual vários hábitos e comportamentos são estabelecidos, incorporados e, possivelmente, transferidos à idade adulta, tornando-se mais difíceis de serem alterados(16).

Com base neste panorama, este estudo objetivou avaliar o NAF de adolescentes, bem como investigar sua associação com variáveis sociodemográficas.

\section{MÉTODOS}

\section{População e Amostra}

O presente estudo foi desenvolvido com base no banco de dados do projeto de pesquisa "Análise da qualidade de vida, prevalência do consumo de álcool, nível de atividade física e índice de massa corporal em estudantes do ensino médio da cidade da Lapa-PR", realizado de junho a novembro de 2005. Os protocolos do estudo foram aprovados pelo Comitê de Ética em Pesquisa da Universidade Estadual de Ponta Grossa, Paraná (Processo no 020/2005).

O projeto envolveu estudantes de 14 a 20 anos de idade, de ambos os sexos, regularmente matriculados na rede pública e particular do ensino médio do município da Lapa, no Estado do Paraná, Região Sul do Brasil. O município é considerado como integrante da região metropolitana de Curitiba, capital do estado, e distante a aproximadamente $70 \mathrm{Km}$. A população do município no ano de 2005 foi estimada em 44733 habitantes. A Rede de Ensino contava com 1596 estudantes regularmente matriculados do $1^{\text {a }}$ ao $3^{\text {a }}$ ano do ensino médio, distribuídos em sete escolas: duas da rede particular $(n=75)$; duas públicas de área urbana $(n=1.183)$ e três públicas de área rural $(n=338)$. A perspectiva do estudo era avaliar todos os adolescentes matriculados no ensino médio. No entanto, a amostra final incluiu 608 sujeitos entre 14 e 20 anos de idade, que tiveram todos os dados tabulados e que participaram voluntariamente, representando $38,1 \%$ da população de estudantes do ensino médio, subdivididos nos seguintes estratos: Escolas Particulares, 24 adolescentes (representando 32\% desse estrato); Escolas Públicas da área Urbana, 391 
adolescentes (33,1\% do estrato); Escolas Públicas da área Rural, 193 adolescentes (57,1\% do estrato). Desta forma, visando confirmar a representatividade da amostra investigada em relação à população de estudo, foi calculado o erro amostral para prevalência de sedentarismo de acordo com os critérios estabelecidos por Gil(7). O erro amostral encontrado $(0,7 \%)$ foi inferior a $1 \%$, valor considerado excelente e garantia da representatividade da amostra( ${ }^{(7)}$.

\section{Instrumentos e Procedimentos}

Primeiramente, foi enviado aos pais e/ou responsáveis dos adolescentes o Termo de Consentimento Livre e Esclarecido (TCLE). Com a devolução do TCLE devidamente assinado, o adolescente era incluído na amostra para a subseqüente avaliação. Foram investigadas variáveis referentes ao NAF e fatores sociodemográficos (tipo de escola, local de moradia, sexo, condição socioeconômica, idade e turno de estudo).

O NAF foi mensurado através do Questionário Internacional de Atividade Física (International Physical Activity Questionnaire - IPAQ; versão 8, forma curta, última semana), desenvolvido pela OMS, com versão em Português validada por Matsudo et al.(19). Recentemente, o IPAQ (versão 8) foi validado para adolescentes por Guedes et al.(9), e os resultados do estudo mostram que, em adolescentes de ambos os sexos com mais de 14 anos, o IPAQ apresenta aceitáveis propriedades de medida para monitorar o NAF. Para classificação da atividade física dos adolescentes foi utilizado o critério desenvolvido pelo Comitê de Pesquisas sobre o IPAQ(15). Esta classificação leva em consideração os critérios de freqüência e duração, e classifica as pessoas em três categorias: inativo; moderadamente ativo e muito ativo. Além da classificação desenvolvida pelo Comitê de Pesquisas sobre o IPAQ, também foi utilizada, com finalidade estatística para análise de regressão logística, a técnica de categorização da amostra por meio de quartis.

A condição socioeconômica foi avaliada de acordo com o Critério de Classificação Econômica do Brasil, desenvolvido pela Associação Brasileira de Empresas de Pesquisa(2). Para este estudo, as classes socioeconômicas foram agrupadas em classe alta $\mathrm{A} 1+\mathrm{A} 2$, classe média $\mathrm{B} 1+\mathrm{B} 2$ e classe baixa $\mathrm{C}+\mathrm{D}+\mathrm{E}$, com intuito estatístico.

\section{Análise Estatística}

Inicialmente, recorreu-se a análise descritiva do NAF dos adolescentes estudados. Em seguida, foi realizado o teste de Kolmogorov-Smirnov para analisar a normalidade da variável atividade física com base nos valores do gasto energético (MET-minuto/semana). Tendo em vista que os dados não apresentaram normalidade, optou-se pela transformação logarítmica para utilização de testes paramétricos. Desta forma, foram investigadas diferenças para o NAF entre os seguintes grupos: sexo, idade, condição socioeconômica, tipo de escola, local de moradia e turno de estudo. Para as variáveis tipo de escola, local de moradia e sexo foi utilizado o teste t de Student para amostras independentes e ANOVA one-way para as variáveis condição socioeconômica e idade. Para analisar diferenças percentuais entre os grupos supracitados para as classificações do IPAQ foi empregado o teste do Qui-quadrado. A Regressão Logística binária (análise bruta e ajustada) foi usada para examinar as associações entre variáveis sociodemográficas (variáveis independentes) com o NAF (variável dependente) dos adolescentes. O nível de significância foi fixado em $\mathrm{p}<0,05$.

\section{RESULTADOS}

O NAF da maioria dos adolescentes investigados foi classificado como alto $(76,8 \%)$ ou moderado (20,9\%) e apenas $2,3 \%$ apresentaram NAF baixo (Tabela 2), com gasto energético médio de 7039 \pm 5602 MET-minuto/semana (tabela 1).

Adolescentes de escolas públicas apresentaram maior gasto energético do que seus pares de escolas particulares $(t=1,643 ; p=0,050)$, bem como moradores da área rural mostraram-se mais ativos do que adolescentes da área urbana $(\mathrm{t}=2,403$; $\mathrm{p}=0,009)$. Além disso, adolescentes do sexo masculino apresentaram maior gasto energético proveniente da prática de atividades físicas do que adolescentes do sexo feminino $(t=3,682 ; p=0,001)$. Não foram observadas diferenças entre adolescentes pertencentes a diferentes condições socioeconômicas ( $\mathrm{A}, \mathrm{B}$ e $\mathrm{C}+\mathrm{D}+\mathrm{E})$, bem como o gasto energético foi semelhante entre todas as idades analisadas, 14 a 20 anos (Tabela 1). 
Tabela 1. Atividade física dos adolescentes (METs-minuto/semana) com valores expressos em média e desvio padrão para a amostra total e estratificado por tipo de escola, local de moradia, sexo, condição socioeconômica e idade.

\begin{tabular}{|c|c|c|c|}
\hline \multirow[t]{2}{*}{ Amostra Estudada } & \multicolumn{3}{|c|}{$\begin{array}{c}\text { Atividade Física } \\
\text { (METs-minuto/semana) }\end{array}$} \\
\hline & Média & Desvio Padrão & $\mathrm{p}^{*}$ \\
\hline Amostra total & 7039 & 5602 & - \\
\hline \multicolumn{4}{|l|}{ Tipo de Escola } \\
\hline Pública & 7115 & 5663 & 0,050 \\
\hline Particular & 5201 & 3390 & \\
\hline \multicolumn{4}{|l|}{ Local de Moradia } \\
\hline Área Rural & 7909 & 6501 & 0,009 \\
\hline Área Urbana & 6634 & 5088 & \\
\hline \multicolumn{4}{|l|}{ Sexo } \\
\hline Masculino & 8130 & 6469 & 0,001 \\
\hline Feminino & 6332 & 4839 & \\
\hline
\end{tabular}

\section{Condição Socioeconômica}

\begin{tabular}{llll} 
A & 5718 & 2992 & 0,380 \\
B & 6981 & 6039 & \\
C, De E & 7161 & 5569 & \\
\hline
\end{tabular}

\begin{tabular}{llll}
\hline Idade & & & \\
14 & 7660 & 5606 & 0,928 \\
15 & 7138 & 5462 & \\
16 & 6916 & 5503 & \\
17 & 6851 & 5789 & \\
18 & 6667 & 6331 & \\
19 & 7386 & 5649 & \\
20 & 5382 & 5925 & \\
\hline
\end{tabular}

* Nível de significância do Teste t de Student para amostras independentes e Anova One-Way.

A classificação do NAF dos adolescentes em relação ao tipo de escola, local de moradia, sexo, condição socioeconômica e idade pode ser observada na tabela 2. Não foram observadas diferenças de acordo com o teste do Qui-quadrado para a proporção de adolescentes com NAF alto, moderado e baixo para nenhuma das variáveis sociodemográficas analisadas.
Tabela 2. Classificação do nível de atividade física dos adolescentes para a amostra total e estratificado por variáveis sociodemográficas de acordo com critério desenvolvido pelo Comitê de Pesquisas sobre o IPAQ(15).

\begin{tabular}{lccc}
\hline Amostra Estudada & \multicolumn{3}{c}{ Classificação do NAF\# } \\
& Alto & Moderado & Baixo \\
\hline Amostra total & 76,8 & 20,9 & 2,3 \\
\hline Tipo de Escola & 77,1 & 20,7 & 2,2 \\
Pública & 70,8 & 25,0 & 4,2 \\
Particular & & & \\
\hline Local de Moradia & & & \\
Área Rural & 80,8 & 16,6 & 2,6 \\
Área Urbana & 75,0 & 22,8 & 2,2 \\
\hline
\end{tabular}

\section{Sexo}

\begin{tabular}{llll} 
Masculino & 78,6 & 19,7 & 1,7 \\
Feminino & 75,7 & 21,6 & 2,7 \\
\hline
\end{tabular}

\section{Condição Socioeconômica}

\begin{tabular}{lccc} 
A & 83,9 & 12,9 & 3,2 \\
B & 71,8 & 24,5 & 3,7 \\
C, D e E & 78,3 & 20 & 1,7 \\
\hline Idade & & & \\
14 & 79,5 & 17,8 & 2,7 \\
15 & 77,2 & 19,1 & 3,7 \\
16 & 77,3 & 21,1 & 1,6 \\
17 & 78,2 & 19,8 & 2,0 \\
18 & 70,5 & 29,5 & 0 \\
19 & 72,7 & 27,3 & 0 \\
20 & 50 & 50 & 0 \\
\hline
\end{tabular}

Os resultados referentes à associação entre a atividade física em adolescentes e as variáveis sociodemográficas investigadas estão descritos na tabela 3. Levando-se em consideração a análise bruta, adolescentes do sexo feminino tiveram 1,63 vezes mais chance de apresentar NAF baixo quando comparadas com adolescentes do sexo masculino $(\mathrm{RC}=1,63$; IC 95\% 1,17-2,26; $\mathrm{p}=0,004)$, bem como adolescentes estudantes no turno matutino tiveram 2,17 vezes 
mais chance de apresentar NAF baixo em relação a seus pares do turno noturno ( $\mathrm{RC}=2,17$; IC $95 \%$ $1,37-3,44 ; \mathrm{p}=0,003$ ).

Com base nos resultados da análise ajustada, podese observar que as variáveis que permaneceram significativas foram sexo $(\mathrm{RC}=1,63$; IC $95 \% 1,17-2,27$; $\mathrm{p}=0,004)$ e turno de estudo ( $\mathrm{RC}=2,12$; IC $95 \%$ 1,34-3,37; $\mathrm{p}=0,003$ ), sendo que adolescentes do sexo feminino e estudantes do turno matutino foram os grupos com maior risco para apresentar NAF baixo. Para as variáveis sociodemográficas tipo de escola, local de moradia, condição socioeconômica e idade as associações com a atividade física não foram significativas.

\section{DISCUSSÃO}

Os achados para a prática habitual de atividade física indicaram que, quando comparados com outros adolescentes (17, 22, 28,2 9), a amostra estudada apresentou resultados satisfatórios, tanto em relação à quantidade de indivíduos classificados como NAF baixo $(2,3 \%)$ quanto ao valor médio do MET-minuto/semana (7039).

Sabe-se que existem inúmeras diferenças em relação aos procedimentos metodológicos utilizados, tanto em relação ao desenvolvimento dos instrumentos de medida da atividade física quanto aos critérios de classificação para determinar o $\operatorname{NAF}^{(13)}$. Desta forma, a comparação entre resultados de estudos que avaliam o NAF através de diferentes métodos, ou então, por meio do mesmo método, porém com instrumentos diferentes, deve ser analisada com cautela.

Com base nas diferenças observadas entre o presente estudo e outras pesquisas, duas hipóteses podem ser levantas: a) os adolescentes do município da Lapa, Paraná, superestimaram seu NAF; b) estas diferenças podem estar relacionadas a diferenças ambientais, tais como: infra-estrutura para realização de atividades de lazer ativo (parques, clubes, quadras esportivas e ciclovias), segurança, clima e tamanho da cidade. Neste sentido, cabe ressaltar que a cidade da Lapa é considerada de pequeno porte, possibilitando o deslocamento a pé ou de bicicleta para qualquer região da cidade, possui um parque com estrutura razoável para prática de atividades físicas, possui inúmeras quadras poliesportivas (praticamente uma
Tabela 3. Associação entre a atividade física e variáveis sociodemográficas em adolescentes.

\begin{tabular}{lccc}
\hline Variáveis & $\mathrm{NAF}^{\#}$ & Análise & Análise \\
[Categorias $]$ & baixo & bruta & ajustada*** \\
& $\%$ & $\mathrm{RC}[95 \% \mathrm{IC}]$ & $\mathrm{RC}(95 \% \mathrm{IC}]$ \\
\hline
\end{tabular}

Tipo de Escola

\begin{tabular}{|c|c|c|c|}
\hline Pública & 49,3 & 1 & 1 \\
\hline Particular & 66,7 & $2,06(0,87-4,88)$ & $1,61(0,66-3,91)$ \\
\hline \multicolumn{4}{|c|}{ Local de Moradia } \\
\hline Área Rural & 45,6 & 1 & 1 \\
\hline Área Urbana & 52,0 & $1,30(0,92-1,82)$ & $1,12(0,78-1,61)$ \\
\hline \multicolumn{4}{|l|}{ Sexo } \\
\hline Masculino & 42,7 & $1^{*}$ & $1^{* *}$ \\
\hline Feminino & 54,7 & $1,63(1,17-2,26)$ & $1,63(1,17-2,27)$ \\
\hline \multicolumn{4}{|c|}{ Condição Socioeconômica } \\
\hline A & 58,1 & $1,48(0,71-3,10)$ & $1,14(0,48-2,70)$ \\
\hline B & 52,8 & $1,20(0,83-1,72)$ & $1,14(0,77-1,70)$ \\
\hline$C, D, E$ & 48,3 & 1 & 1 \\
\hline \multicolumn{4}{|l|}{ Idade } \\
\hline 14 & 43,8 & 1 & 1 \\
\hline 15 & 51,6 & $1,37(0,79-2,35)$ & $1,47(0,84-2,56)$ \\
\hline 16 & 48,6 & 1,21 (0,70-2,09) & $1,34(0,77-2,34)$ \\
\hline 17 & 52,5 & $1,42(0,77-2,59)$ & $1,71(0,92-3,19)$ \\
\hline 18 & 54,5 & $1,54(0,73-3,26)$ & $2,12(0,97-4,63)$ \\
\hline 19 & 36,4 & $0,73(0,20-2,72)$ & $0,92(0,24-3,49)$ \\
\hline 20 & 66,7 & $2,56(0,44-14,88)$ & $3,74(0,63-22,08)$ \\
\hline
\end{tabular}

Turno de Estudo

\begin{tabular}{llcc} 
Matutino & 56,0 & $2,17(1,37-3,44)$ & $2,12(1,34-3,37)$ \\
Vespertino & 47,8 & $1,57(0,96-2,54)$ & $1,45(0,89-2,37)$ \\
Noturno & 36,9 & $1^{*}$ & $1^{* *}$ \\
\hline
\end{tabular}

${ }^{*} p<0,05$ para a análise bruta; ${ }^{* *} p<0,05$ para a análise ajustada. ****Análise ajustada para o tipo de escola, local de moradia, sexo, condição socioeconômica, idade e turno de estudo; \#NAF = Nível de Atividade Física

em cada bairro da cidade), contempla um índice muito baixo de criminalidade, bem como, trânsito de automóveis reduzido e tem um clima agradável para prática de exercícios físicos. O somatório destes 
fatores pode ser o responsável pelo alto NAF observado para a amostra investigada na presente pesquisa. Em relação a diferenças para as categorias do NAF mediadas por fatores sociodemográficos, os resultados indicaram que as variáveis tipo de escola, local de moradia, sexo, condição socioeconômica e idade parecem não interferir na atividade física da amostra investigada. Entretanto, quando os dados do MET são analisados, observa-se que adolescentes de escolas públicas possuem gasto energético superior aos estudantes em escolas particulares, da mesma forma, moradores da área rural são mais ativos que seus pares da área urbana, bem como rapazes apresentam maior gasto energético do que moças. Estes achados demonstram que a categorização da variável atividade física pode maquiar alguns resultados importantes para determinação de grupos mais propensos a apresentar baixo gasto energético.

As diferenças observadas no presente estudo são consistentes com achados de outras investigações.

Grande parte das pesquisas nacionais e internacionais que compararam a atividade física entre rapazes e moças constatou que adolescentes do sexo masculino foram mais ativos quando comparados com o sexo feminino(11,12,24,27). Estes achados conduzem à reflexão de que estratégias de intervenção devem ser direcionadas ao público feminino, principalmente durante a adolescência, tendo em vista que há indícios consistentes, suportados por estudos longitudinais, de que o NAF sofre uma diminuição drástica na transição da infância para a adultícia, em especial em meninas $^{(14,25)}$. No entanto, cabe ressaltar que no presente estudo não foi observado diferença para o NAF entre adolescentes mais jovens e mais velhos.

Poucas pesquisas vêm sendo realizadas visando comparar o NAF de adolescentes estudantes em escolas públicas e particulares. Desta forma, comparações dos achados da presente pesquisa com outras investigações ficam prejudicadas. Um dos fatores que poderia explicar contrastes no gasto energético de estudantes de escolas públicas e particulares seria a condição socioeconômica, no entanto, não foram observadas diferenças para o NAF de adolescentes pertencentes a diferentes classes econômicas. Neste sentido, parece que outras variáveis, não mensuradas no presente estudo, podem ser as responsáveis por um maior gasto energético de estudantes em escolas públicas em relação a adolescentes de escolas particulares, indicando a necessidade de investigações mais aprofundadas em relação a este fenômeno. Quanto à influência do local de moradia sobre a atividade física, acredita-se que adolescentes residentes em áreas rurais ajudam seus pais no trabalho do campo durante o período extra-escolar (agricultura, horticultura, pecuária, suinocultura, etc.), enquanto adolescentes da área urbana destinam seu tempo livre para prática de atividades sedentárias, tais como, assistir TV, jogar vídeo-game e navegar na internet. Outros estudos também indicaram que adolescentes rurais parecem apresentar NAF superior aos residentes em área urbana ${ }^{(21,23)}$.

Em relação à análise de regressão logística ajustada, os resultados indicaram o sexo e o turno de estudo como variáveis determinantes da atividade física, sendo que adolescentes do sexo feminino e estudantes do turno matutino foram os grupos com maior risco para apresentar NAF baixo. Quanto à relação entre o NAF e o sexo, como já citado anteriormente, está bem documentado na literatura que, em geral, moças são menos ativas do que rapazes. Estes achados conduzem à inferência de que programas de fomento à prática de atividade física durante a adolescência precisam focar no público feminino, visando atender a uma parcela da população que não está vivenciando os benefícios biopsicossociais da prática de atividades físicas e tende a apresentar, futuramente, problemas à saúde relacionados ao sedentarismo. Estas estratégias devem ser desenvolvidas com base no incentivo à prática de exercícios entre moças e, principalmente, através do suporte às adolescentes para realização de atividades preferidas por este estrato da população.

Os motivos para que moças apresentem um NAF menor do que rapazes são diversos e podem estar relacionados a aspectos sociais, econômicos e culturais. Estudo desenvolvido por Gonçalves et al.(8), com o objetivo de avaliar o efeito de variáveis socioculturais sobre o NAF, através de uma criativa combinação entre duas abordagens metodológicas (epidemiológica e etnográfica) forneceu dados importantes sobre os motivos que levam moças a serem menos ativas do que rapazes. Os resultados do estudo indicaram que rapazes têm mais apoio social e familiar para a realização de atividades físicas na adolescência do 
que moças. Os autores acreditam que historicamente a socialização e a educação de moças e rapazes se diferenciaram, sendo que moças tendem a ser mais vigiadas e impostas a algumas exigências culturais que podem ajudar a explicar o menor NAF em comparação aos rapazes.

Quanto à associação entre NAF e turno de estudo, observou-se que o principal subgrupo populacional em risco para o sedentarismo foi composto por estudantes do período matutino. Estes achados foram semelhantes aos observados por Tenório et al. (2010)(30) em adolescentes estudantes da rede pública estadual de ensino médio do Estado de Pernambuco, Brasil. Contudo, Tenório et al.(30) não conduziram a discussão na tentativa de explicitar os motivos que poderiam explicar a relação entre o turno de estudo e a inatividade física em adolescentes. Embora haja carência de estudos que possam auxiliar no entendimento deste fenômeno, o menor NAF observado para estudantes do período matutino é um achado um tanto quanto surpreendente. Estes adolescentes, em geral, têm mais tempo livre para a prática de atividades físicas, pois, na maioria dos casos, não trabalham. Entretanto, algumas questões podem ser levantadas na tentativa de elucidar porque adolescentes do turno matutino apresentaram maior risco para possuir NAF baixo, tais como: deslocamento até o colégio por meio de automóvel, pouco tempo de permanência ao ar livre e atividades sedentárias no tempo livre (extra-escolar). Além disso, pode-se especular que adolescentes estudantes do turno noturno tenham apresentado NAF superior a estudantes do período matutino devido a engajamento em atividades laborais pesadas, no entanto, a variável trabalho não foi mensurada e, desta forma, estas argumentações devem ser analisadas com cautela.

Com base neste contexto, parece ser necessário o incentivo a prática de atividades físicas no período de tempo livre entre adolescentes estudantes no turno matutino, bem como se faz necessário oportunizar aos adolescentes o engajamento em programas de atividades físicas regulares. Nesta perspectiva, o ambiente escolar apresenta-se como um meio propício para que atividades coordenadas por professores de Educação Física possam ser desenvolvidas durante o tempo extra-escolar, visando aumentar o
NAF de adolescentes estudantes no turno matutino. As variáveis sociodemográficas não modificáveis sugerem subgrupos de pessoas jovens relativamente inativas que precisam ser alvo para programas de tratamento. Neste sentido, investigações futuras precisam ser realizadas, visando mapear grupos que apresentem maiores dificuldades para realização de atividades físicas, bem como trabalhos de intervenção devem ser conduzidos considerando diferenças sociodemográficas entre adolescentes. As principais contribuições do presente estudo foram: a) o avanço do conhecimento sobre o NAF de adolescentes de uma cidade de pequeno porte da região metropolitana da capital do estado do Paraná, tendo em vista que dados sobre esta região são pouco documentados na literatura; e b) a determinação de variáveis sociodemográficas determinantes do NAF de adolescentes, especialmente o turno de estudo, uma variável que precisa ser considerada e mais bem explorada em investigações futuras. Com base nos achados do presente estudo, pode-se inferir que a proporção de adolescentes que apresentaram NAF baixo foi bastante reduzida (2,3\%), sendo que moças e estudantes do turno matutino foram os grupos com maior risco para apresentar este desfecho. Neste sentido, programas de promoção da saúde e incentivo à prática de atividade física regular, devem considerar o sexo e o turno de estudo como variáveis chave para mudanças no NAF de adolescentes.

\section{CORRESPONDÊNCIA}

\section{Alex Pinheiro Gordia}

Centro de Pesquisa em Exercício e Esporte,

Departamento de Educação Física, Universidade

Federal do Paraná,

Rua Coração de Maria, 92, 80215-370,

Curitiba, Paraná

E-mail: alexgordia@gmail.com 


\section{REFERÊNCIAS}

1. Andersen LB, Harro M, Sardinha LB, Froberg K, Ekelund U, Brage S, Anderssen SA (2006). Physical activity and clustered cardiovascular risk in children: a cross-sectional study (The European Youth Heart Study). Lancet, 368 (9532): 299-304

2. Associação Brasileira de Empresas de Pesquisa (2003). Dados com base no Levantamento Sócio Econômico, 2000 IBOPE. http://www.abep.org 10/05/2005.

3. Berenson GS (2002). Childhood risk factors predict adult risk associated with subclinical cardiovascular disease. The Bogalusa Heart Study. Am J Cardiol, 90(10): 3-7

4. Berenson GS, Srnivasan SR, Bogalusa Heart Study Group (2005). Cardiovascular risk factors in youth with implications for aging: the Bogalusa Heart Study. Neurobiol Aging, 26(3): 303-307

5. Boscolo RA, Sacco IC, Antunes HK, Mello MT, Tufik S (2007). Avaliação do padrão de sono, atividade física e funções cognitivas em adolescentes escolares. Rev Port Cien Desp, 7(1): 18-25

6. Faria Júnior JC, Nahas MV, Barros MVG, Loch MR, Oliveira ESA, De Bem MFL, Lopes AS (2009).

Comportamentos de risco à saúde em adolescentes no Sul do Brasil: prevalência e fatores associados. Rev Panam Salud Publica, 25(4): 344-352

7. Gil AC (1991). Métodos e técnicas de pesquisa social. (3 $\left.3^{\mathrm{a}} \mathrm{ed}.\right)$, São Paulo: Atlas.

8. Gonçalves H, Hallal PC, Amorim TC, Araújo CLP, Menezes AMB (2007). Fatores socioculturais e nível de atividade física no início da adolescência. Rev Panam Salud Publica, 22(4): 246-253

9. Guedes DP, Lopes CC, Guedes JERP (2005).

Reprodutibilidade e validade do Questionário Internacional de Atividade Física em adolescentes. Rev Bras Med Esporte, 11(2): 151-158

10. Hallal PC, Victora CG, Azevedo MR, Wells JCK (2006). Adolescent physical activity and health. Sports Med, 36(12): 1019-1030

11. Hallal PC, Bertoldi AD, Gonçalves H, Victora CG (2006). Prevalência de sedentarismo e fatores associados em adolescentes de 10-12 anos de idade. Cad Saúde Pública, 22(6): 1277-1287

12. Hallal PC, Wells JCK, Reichert FF, Anselmi L, Victora CG (2006). Early determinants of physical activity in adolescence: prospective birth cohort study. BMJ, 332(7548): 1002-1014

13. Hallal PC, Dumith SC, Bastos JP, Reichert FF, Siqueira FV, Azevedo MR (2007). Evolução da pesquisa epidemiológica em atividade física no Brasil: revisão sistemática. Rev Saúde Pública, 41(3): 453-460

14. Harrell JS, Mcmurray RG, Baggett CD, Pennell ML, Pearce PF, Bangdiwala SI (2005). Energy costs of physical activities in children and adolescents. Med Sci Sports Exerc, 37(2): 329-336

15. Ipaq Research Committee (2005). Guidelines for Data Processing and Analysis of the International Physical Activity Questionnaire (IPAQ). http://www.ipaq.ki.se/ 19/05/2007.

16. Kim SYS, Kwiterovich PO (1995). Childhood prevention of adults chronic diseases: rationale and strategies. In: Cheung LWY, Richmond JB, (Editors), Child health, nutrition, and physical activity. Champaign, IL: Human Kinetics

17. Koezuka N, Koo M, Allison KR, Adlaf EM, Dwyer JJM,
Faulkner G, Goodman J (2006). The relationship between sedentary activities and physical inactivity among adolescents: results from the Canadian Community Health Survey. J Adolesc Health, 39(4): 515-522

18. Kriska AM, Brach JS, Jarvis BJ, Everhart JE, Fabio A, Richardson CR, Howard BV (2007). Physical activity and gallbladder disease determined by ultrasonography. Med Sci Sports Exerc, 39(11): 1927-1932

19. Matsudo SM, Araújo T, Matsudo VR, Andrade D, Andrade E, Oliveira LC, Braggion G (2001). Questionário Internacional de Atividade Física (IPAQ): Estudo de validade e reprodutibilidade no Brasil. Rev Bras Ativ Fís Saúde, 6(2): 5-8

20. Mora S, Cook N, Buring JE, Ridker PM, Lee IM (2007). Physical activity and reduced risk of cardiovascular events: potential mediating mechanisms. Circulation, 116(19): 2110-2118

21. Nelson MC, Gordon-Larsen P, Song Y, Popkin BM (2006). Built and social environments associations with adolescent overweight and activity. Am J Prev Medicine, 31(2): 109-117

22. Oehlschlaeger MHK, Pinheiro RT, Horta B, Gelatti C, San'tana P (2004). Prevalência e fatores associados ao sedentarismo em adolescentes de área urbana. Rev Saúde Pública, 38(2): 157-163

23. Özdirenç M, Özcan A, Akin F, Gelecek N (2005). Physical fitness in rural children compared with urban children in Turkey. Pediatr Int, 47(1): 26-31

24. Platat C, Wagner A, Klumpp T, Schweitzer B, Simon C (2006). Relationships of physical activity with metabolic syndrome features and low-grade inflammation in adolescents. Diabetologia, 49(9): 2078-2085

25. Raustorp A, Mattsson E, Svensson K, Stahle A (2006). Physical activity, body composition and physical selfesteem: a 3-year follow-up study among adolescents in Sweden. Scand J Med Sci Sports, 16(4): 258-266

26. Rodearmel SJ, Wyatt HR, Stroebele N, Smith SM, Ogden LG, Hill JO (2007). Small changes in dietary sugar and physical activity as an approach to preventing excessive weight gain: The America on the Move family study. Pediatrics, 120(4): 869-879

27. Shi Z, Lien N, Kumarb BN, Holmboe-Ottesen G (2006). Physical activity and associated socio-demographic factors among school adolescents in Jiangsu Province, China. Prev Medicine, 43(3): 218-221

28. Silva GSF, Bergamaschine R, Rosa M, Melo C, Miranda R, Bara Filho M (2007). Avaliação do nível de atividade física de estudantes de graduação das áreas saúde/biológica. Rev Bras Med Esporte, 13(1): 39-42

29. Silva RCR, Malina RM (2000). Nível de atividade física em adolescentes do Município de Niterói, Rio de Janeiro, Brasil. Cad Saúde Pública, 16(4):1091-1097

30. Tenório MCM, Barros MVG, Tassitano RM, Bezerra J, Tenório JM, Hallal PC (2010). Atividade física e comportamento sedentário em adolescentes estudantes do ensino médio. Rev Bras Epidemiol, 13(1): 105-117

31. Wolin KY, Lee IM, Colditz GA, Glynn RJ, Fuchs C, Giovannucci E (2007). Leisure-time physical activity patterns and risk of colon cancer in women. Int $J$ Cancer, 121(12): 2776-2781

32. Wolin KY, Glynn RJ, Colditz GA, Lee IM, Kawachi I (2007). Long-term physical activity patterns and healthrelated quality of life in U.S. women. Am J Prev Med, 32 (6): 490-499 